\title{
Highly Sensitive Reentrant Cavity-Microstrip Patch Antenna Integrated Wireless Passive Pressure Sensor for High Temperature Applications
}

\author{
Fei Lu, ${ }^{1,2}$ Yanjie Guo, ${ }^{1,2}$ Qiulin Tan, ${ }^{1,2}$ Tanyong Wei, ${ }^{1,2}$ Guozhu Wu, ${ }^{1,2}$ Haixing Wang, ${ }^{1,2}$ \\ Lei Zhang, ${ }^{1,2}$ Xiaowei Guo, ${ }^{1,2}$ and Jijun Xiong ${ }^{1,2}$ \\ ${ }^{1}$ Key Laboratory of Instrumentation Science \& Dynamic Measurement, Ministry of Education, North University of China, \\ Taiyuan 030051, China \\ ${ }^{2}$ Science and Technology on Electronic Test and Measurement Laboratory, North University of China, Taiyuan 030051, China \\ Correspondence should be addressed to Qiulin Tan; tanqiulin@nuc.edu.cn
}

Received 23 May 2017; Accepted 25 September 2017; Published 19 October 2017

Academic Editor: Guiyun Tian

Copyright (C) 2017 Fei Lu et al. This is an open access article distributed under the Creative Commons Attribution License, which permits unrestricted use, distribution, and reproduction in any medium, provided the original work is properly cited.

\begin{abstract}
A novel reentrant cavity-microstrip patch antenna integrated wireless passive pressure sensor was proposed in this paper for high temperature applications. The reentrant cavity was analyzed from aspects of distributed model and equivalent lumped circuit model, on the basis of which an optimal sensor structure integrated with a rectangular microstrip patch antenna was proposed to better transmit/receive wireless signals. In this paper, the proposed sensor was fabricated with high temperature resistant alumina ceramic and silver metalization with weld sealing, and it was measured in a hermetic metal tank with nitrogen pressure loading. It was verified that the sensor was highly sensitive, keeping stable performance up to $300 \mathrm{kPa}$ with an average sensitivity of $981.8 \mathrm{kHz} / \mathrm{kPa}$ at temperature $25^{\circ} \mathrm{C}$, while, for high temperature measurement, the sensor can operate properly under pressure of $60-120 \mathrm{kPa}$ in the temperature range of $25-300^{\circ} \mathrm{C}$ with maximum pressure sensitivity of $179.2 \mathrm{kHz} / \mathrm{kPa}$. In practical application, the proposed sensor is used in a method called table lookup with a maximum error of $5.78 \%$.
\end{abstract}

\section{Introduction}

Wireless passive sensors are now in urgent research needs for application of extreme conditions, for example, high temperature, high speed of rotation, and high pressure. Like in aerospace, the development of aviation engines and gas turbines property, design evaluation, and performance monitoring are key factors which affect the development of the aerospace industry. For an engine, combustion chamber and turbine are the two core parts which need to survive at temperature up to $2000 \mathrm{~K}$, of which the high temperature property directly affects the working efficiency and life of the engine [1]. By now, the engine inner parameters cannot be measured in situ directly due to the fact that most sensors cannot survive in high temperature for the limitation of materials, and they are often wire-connected to accomplish the whole sensing process. High temperature parameters are generally obtained indirectly via simulation from measurement results in external environment.

There are lots of challenges for sensing devices in the development of engine. Conventional test methods require wire connections between the test controller and each test node and the wires are connected with specific signal disposal circuits, causing problems like wire layout, power supply, electromagnetic interference, transmission range, transmission security, and high temperature resistance. Hence, the wireless and passive sensing devices are in an increasing need for extreme conditions. By now, there are generally three kinds of wireless sensing technology, that is, nearfield inductance sensing (LC) technology, surface acoustic wave (SAW) sensing technology, and microwave scattering (RF) signal reading technology. Fonseca proposed a LC 


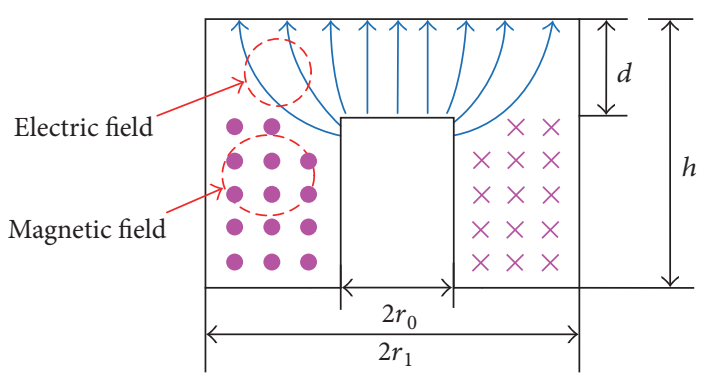

(a)

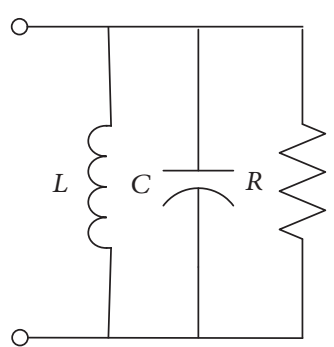

(b)

FIGURE 1: (a) Electromagnetic field distribution of the stimulated reentrant cavity. (b) Equivalent shunt lumped circuit model.

resonance based pressure sensor which operated up to 5 bar in temperature range of $25-400^{\circ} \mathrm{C}$ [2]. It was fabricated in LTCC ceramic with low profile and high integration. Tan et al. proposed LC based sensor which can realize temperature and pressure measurement simultaneously, providing temperature compensation for pressure, making it precise for the measurement of the pressure at high temperature [3]. But the lumped circuit has low $Q$ factor, causing a rapid reduction of the stored energy and a short wireless transmission distance $[4,5]$. For LGS $\left(\mathrm{La}_{3} \mathrm{Ga}_{5} \mathrm{SiO}_{14}\right.$, one of piezoelectric materials) based SAW sensor, it could survive up to $1400^{\circ} \mathrm{C}$, but there are several factors that limit potential use of it, including excessive conductive and viscous losses, deviations from stoichiometry, and chemical instability [6]. Moreover, LGS based sensor has significant loss in the radio frequency spectrum, making it hard to work in high frequency [7], while, for microwave scattering technology, it has advantages of high $Q$ factor and stability in high frequency. In addition, microwave scattering based sensor is not easy to be disturbed by ambient environment for its metal closed structure. Xiong et al. proposed a resonator based wireless temperature sensor which is fabricated in alumina ceramic, realizing temperature measurement up to $800^{\circ} \mathrm{C}$ [4]. But due to the closed cylindrical volume of the sensor, the dimension is not small enough to make it integrated with measured object. Afterwards, Cheng et al. designed a low-profile temperature sensor based on planar patch resonator which can realize temperature measurement up to $1050^{\circ} \mathrm{C}$ [8]. And for pressure measurement, Zhao innovatively proposed a wireless passive sensing technique based on evanescent-mode cavity resonator, successfully demonstrating airflow and displacement measurements by integrating an UWB monopole antenna [9]. But the sensor was not fabricated in high temperature resistant material. Hence, it can just work in normal environment. Cheng et al. fabricated a cylindrical resonator based pressure sensor for wireless pressure measurement up to $5 \mathrm{~N}$ at $1000^{\circ} \mathrm{C}$ [8]. But the pressure was loaded manually via a dielectric rod, not realizing a contactless measurement. It is not suitable for the aforementioned extreme conditions.

Here we proposed a wireless passive pressure sensor composed of a reentrant cavity with an aperture and a rectangular microstrip patch antenna which can operate properly up to $300^{\circ} \mathrm{C}$. It was designed in microwave scattering and fabricated in high temperature resistant alumina ceramic with silver metalization. To verify the validity of the pressure sensor, it was firstly tested in a metal-sealed tank with nitrogen load. It was shown that the sensor was well operated up to 3 bar in $25^{\circ} \mathrm{C}$ with a high average sensitivity of $981.8 \mathrm{kHz} / \mathrm{kPa}$, realizing a contactless measurement for pressure. Then it was tested up to $300^{\circ} \mathrm{C}$ and had a maximum sensitivity of $179.2 \mathrm{kHz} / \mathrm{kPa}$ which was many times higher than the LC based sensor proposed in [10] and SAW based sensor in [11]. It was promising to be used for inner engine pressure measurement in the near future for farther research, realizing a real sense of contactless measurement in harsh environment.

\section{Design, Characterization, and Fabrication of the Sensor}

Reentrant cavity is a tunable microwave resonator which is characterized with a smaller dimension at the same frequency as general cylindrical resonator. For an equivalent cavity model, it is difficult to be accurately calculated using Maxwell's equations. But when the cavity model dimension meets the condition $d \ll h$, as shown in Figure 1(a), and the geometric dimension is smaller than the resonant wavelength, we can assume that the high frequency electric field of the cavity dominant mode $\mathrm{TM}_{010}$ is mainly distributed among the space with height $d$, while the magnetic field is distributed around the embedded cylinder which presents annular concentration [12-14] as shown in Figure 1(a). So we obtain the equivalent lumped circuit model in electrostatic field by analysis of the distributed model according to [15]. The equivalent shunt circuit model is shown in Figure 1(b). $L$ is the equivalent annular inductor and $C$ is the equivalent capacitor which is composed of two parts $C_{0}$ and $C_{1} . C_{0}$ is the capacitance between the inner post top and the center section of the cap which is a parallel-plate capacitor, while $C_{1}$ is the capacitance between the post side wall and the end plate. $R$ is the equivalent resistor of the cavity.

$C_{0}$ can be calculated according to the parallel-plate capacitor expression as

$$
C_{0}=\frac{\varepsilon_{0} \pi r_{0}^{2}}{d}
$$

where $\varepsilon_{0}$ is the permittivity in vacuum, denoting an approximate value of $8.854 \times 10^{-12} \mathrm{~F} / \mathrm{m}, r_{0}$ is the radius of the inner post, and $d$ is the distance between the cap and the top of 


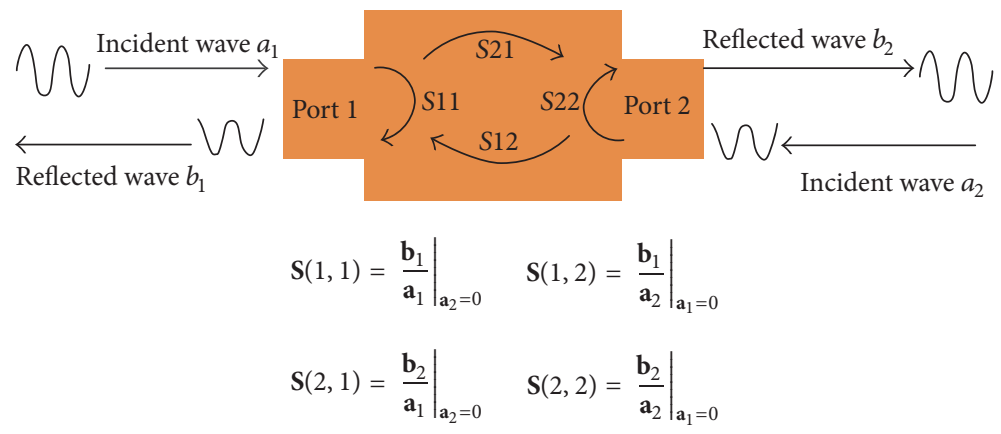

FIgURE 2: Two-port network signal transmission mechanism.

the post. Here we ignore the fringe effect of the parallelplate capacitor for purpose of simplification of the calculation while causing little influence on the analysis. And $C_{1}$ can be expressed according to Green's theorem as [16]

$$
C_{1}=4 \varepsilon_{0} r_{0} \ln \frac{e \sqrt{h^{2}+\left(r_{1}-r_{0}\right)^{2}}}{2 d},
$$

where $r_{1}$ denotes outer radius of the cavity. The equivalent capacitor $C$ of the reentrant cavity is calculated as

$$
C=C_{0}+C_{1} \text {. }
$$

And the equivalent inductor $L$ can be expressed as [16]

$$
L=\frac{\mu_{0} h}{2 \pi} \ln \frac{r_{1}}{r_{0}}
$$

where $\mu_{0}$ is the permeability in vacuum, denoting $4 \pi \times$ $10^{-7} \mathrm{H} / \mathrm{m}$. Here $L$ can be seen as a one-turn annular inductor with cross-section $(h-d) \times\left(r_{1}-r_{0}\right)[16]$.

From the above expressions, (1)-(4), the resonant frequency of the reentrant cavity can be calculated as

$$
\begin{aligned}
f_{0} & =\frac{1}{2 \pi \sqrt{L C}} \\
& =\frac{c}{2 \pi}\left\{r_{0} h\left[\frac{r_{0}}{2 d}-\frac{2}{\pi} \ln \frac{2 d}{e \sqrt{h^{2}+\left(r_{1}-r_{0}\right)^{2}}}\right]\right. \\
& \left.\cdot \ln \frac{r_{1}}{r_{0}}\right\}^{-1 / 2} .
\end{aligned}
$$

Accordingly, the quality factor of reentrant cavity $Q$ is approximately given by [17]

$$
\frac{1}{\mathrm{Q}}=\delta\left(\frac{1}{h}+\frac{(h-d) / r_{0} h+1 / r_{1}}{2 \ln \left(r_{1} / r_{0}\right)}\right),
$$

where $\delta$ denotes skin depth of specific metallic conductor and it is related to resonant frequency $f_{0}$ and can be expressed as

$$
\delta=\frac{2}{\sqrt{2 \pi \mu_{0} \sigma f_{0}}}
$$

where $\sigma$ denotes conductivity of metallic conductor, as for Dupont $6142 \mathrm{D}$ silver used for the fabrication of the sensor in the experiment, it is $6.1 \times 10^{7} \mathrm{~S} / \mathrm{m}$.

It can be known from the above analysis that a reentrant cavity is an all-metal-sealed structure which is sensitive to deformation in section with height $d$, but it cannot realize signal interaction with external wireless interrogation antenna. Thus a rectangular aperture needs to be punched to make the internal electromagnetic wave wirelessly coupled with an interrogation antenna. Because the aperture is small in dimension, it makes little perturbation on the electromagnetic field distribution and the resonant frequency of the cavity dominant resonant mode. This was verified by us via Ansoft HFSS software. When the cavity was wirelessly interrogated with an external antenna, one of the electromagnetic wave components transmitted by the interrogation antenna with the same frequency as the reentrant cavity resonant frequency forms standing wave inside the cavity and gradually oscillates till it disappears, while the electromagnetic wave components in other frequencies were reflected to the antenna. Hence, there is frequency deficiency for the received signal compared with the transmitted signal. By analysis of the $S$ parameters of the interrogation antenna, the cavity resonant frequency can be obtained. As shown in Figure 2, the signal process system can be seen as a two-port network. Here we use $S(1,1)$ parameter of the antenna as an evaluation of the signal strength, and the corresponding frequency where valley lies is the resonant frequency of the reentrant cavity sensor.

To make the cavity preferably receive signals transmitted from the interrogation antenna, a reply antenna was designed. A microstrip patch antenna was integrated on the cavity top covering the couple aperture to act as an "intermediary agent" between the antenna and the cavity. It can receive the interrogation signal from the antenna and in turn transmits the response signal of the sensor to the antenna. Based on above analysis, a reentrant cavitymicrostrip patch antenna integrated pressure sensor structure based on alumina ceramic substrate and silver paste was designed in Figure 3.

For the proposed reentrant cavity structure, pressure sensitive part is the cap, as shown in Figure 3(d). When there is uniformly distributed load imposed on the top surface, the cap is deformed inside the cavity, causing the distance 


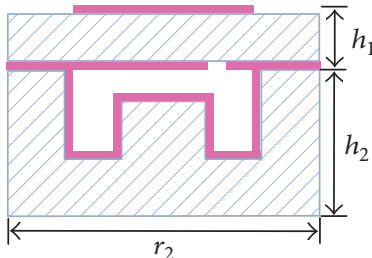

Silver layer Alumina substrate

(a)

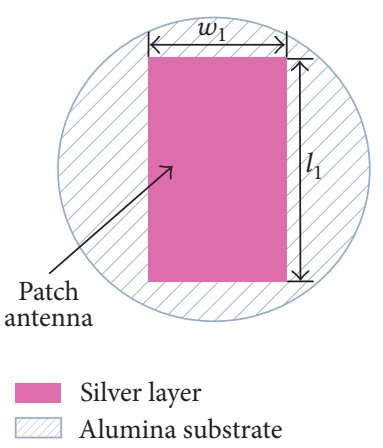

(b)

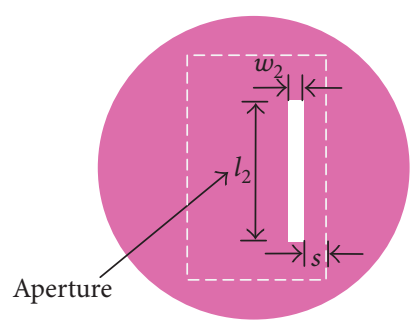

Silver layer

Alumina substrate

(c)

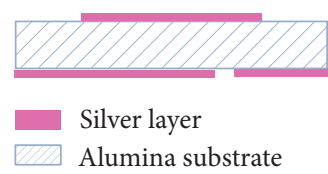

(d)

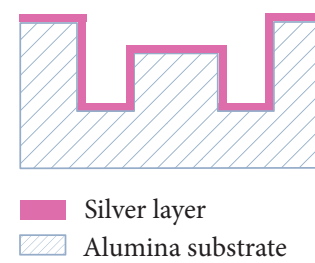

(e)

Figure 3: Mechanical structure of the reentrant cavity sensor. (a) Cross-section view of the assembled sensor. (b) Top view of the sensor cap. (c) Bottom view of the cap. (d) Cross-section view of the cap. (e) Cross-section view of the substrate.

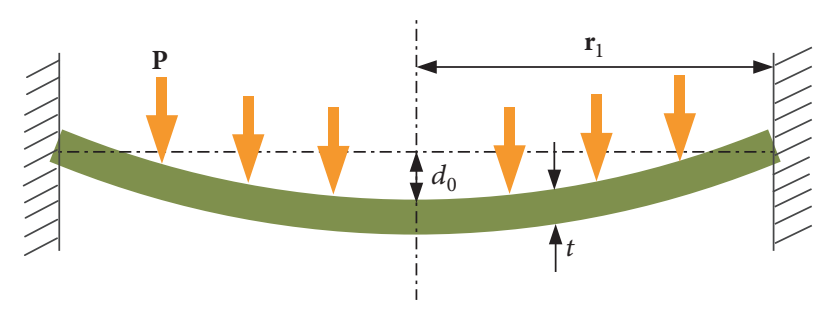

Figure 4: Deformed cap with pressure load.

$d$ to decrease. Thus the equivalent capacitor $C$ of the cavity increases, leading a reduced resonant frequency. We can obtain the measured pressure value via a corresponding relationship between the pressure and sensor resonant frequency. Here the small deflection theory for a round-clamped thin plate was used to analyze the deformation condition of the sensor cap as shown in Figure 4.

The maximum central deformation of the plate can be expressed as [18]

$$
d_{0}=0.01512 \frac{\operatorname{Pr}_{1}^{4}\left(1-v^{2}\right)}{E t^{3}},
$$

where $E$ is Young's modulus of the deformed material, $v$ is Poisson's ratio, and $t$ is the thickness of the cap. For alumina ceramic we used, the specific properties are listed in Table 1.

According to expressions (5) and (8), the sensitivity of the sensor can be calculated as

$$
S(d)=\frac{\partial f}{\partial d}=f_{0}(d)\left(C_{0}^{\prime}(d)+C_{1}^{\prime}(d)\right) .
$$

To determine the sensor dimension and further analyze the sensitivity of the pressure deformable reentrant cavity, we
TABLE 1: Alumina ceramic properties (provided by Bolandi Machinery Equipment Co. Ltd.).

\begin{tabular}{lc}
\hline Property & Value \\
\hline Density $\left(\mathrm{g} / \mathrm{cm}^{3}\right)$ & 3.9 \\
Flexure strength $(\mathrm{MPa})$ & 450 \\
Young's modulus $(\mathrm{GPa})$ & 390 \\
Permittivity & 10 \\
Poisson's ratio & 0.22 \\
\hline
\end{tabular}

preset the sensor model with three different configurations and the relationship between $d$ and resonant frequency was simulated in MATLAB as shown in Figure 5(a). The slope of each curve is seen as $S(d)$. But the deformed cap is a curved surface with maximum deflection in center. It is not exactly in accordance with the flat-cap reentrant cavity. Qualitative analysis was used to evaluate the sensitivity of the sensor and determine the dimension.

It can be seen from Figure 5(a) that the sensor sensitivity is influenced by both the inner post radius $r_{0}$ and $h$. Generally, the resonant frequency variation is more evident under the same pressure when $r_{0}$ and $h$ are smaller, respectively, especially when $d$ is smaller than $0.2 \mathrm{~mm}$. The sensor $Q$ factor variation with $d$ was plotted in Figure 5(b). It can be seen that the $Q$ factor decreases with the decrease of $d$. The tendency is especially distinct when $d$ is smaller than $0.1 \mathrm{~mm}$. For practical measurement, the higher the $Q$ factor is, the further the sensor signal can wirelessly transfer. So there needs to be a tradeoff between the sensor sensitivity and $Q$ factor. The final dimension of the sensor was listed in Table 2.

For the dimension design of the pressure deformable cap, Ansoft ANSYS software was utilized to analyze the deformation and stress of the pressure loaded plate. A circular 


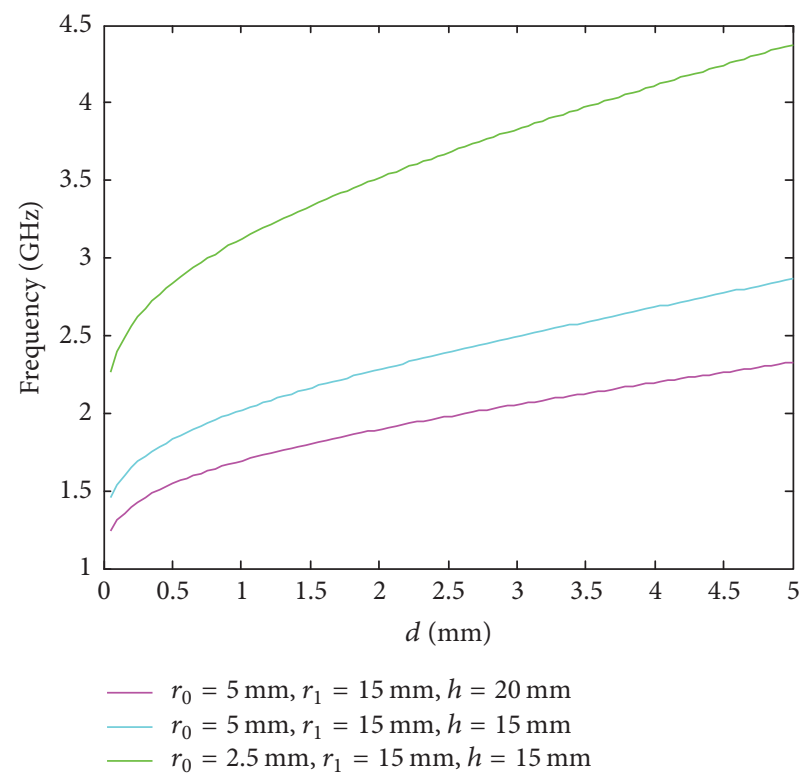

(a)

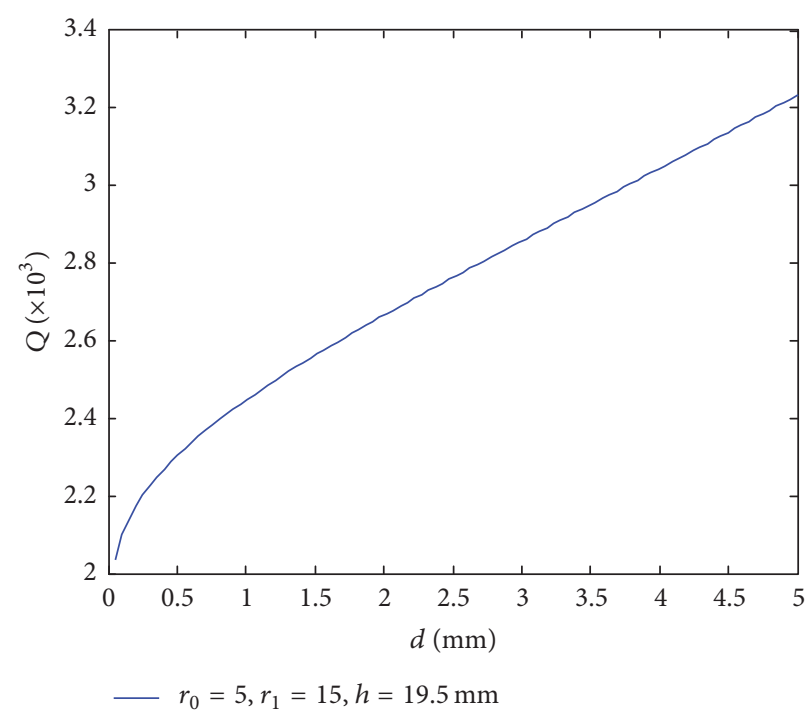

(b)

FIGURE 5: (a) Resonant frequency variation with distance $d$ for three sensor configurations. (b) $Q$ factor variation with $d$.

TABLE 2: Dimension of the sensor ( $\mathrm{mm})$.

\begin{tabular}{lc}
\hline Symbol & Value \\
\hline$r_{0}$ & 5 \\
$r_{1}$ & 15 \\
$h$ & 19.5 \\
$d$ & 1 \\
$r_{2}$ & 20 \\
$h_{2}$ & 24.5 \\
$h_{1}$ & 0.3 \\
$w_{1}$ & 22 \\
$l_{1}$ & 30 \\
$w_{2}$ & 1.5 \\
$l_{2}$ & 22 \\
$s$ & 2 \\
\hline
\end{tabular}

thin plate with radius of $20 \mathrm{~mm}$ and thickness of $0.3 \mathrm{~mm}$ was finally determined as the sensor cap. ANSYS simulation was shown in Figure 6. We can see that the maximum central deflection is $0.949 \mathrm{~mm}$ when the plate is loaded with $3.5 \mathrm{bar}$ uniform pressure, and the maximum stress occurred along the circumference of the cap which is $344 \mathrm{MPa}$. It is smaller than the alumina allowable flexure strength of $450 \mathrm{MPa}$. Hence, the sensor can be operated in a safe pressure range of 0-3.5 bar.

For fabrication of the sensor, a sealed cavity with metalization inside the ceramic wall is needed. It is hard to be realized with existing processing craft. So we fabricated the sensor for separated two parts, that is, the cap and substrate, and then bonded them with welding method. Specific fabrication processes are shown in Figure 7. Firstly, a substrate mould was fabricated in advance for shaping of liquid alumina precursor. A precursor is generally composed of organic monomer, crosslinking agent, dispersant, initiator, and catalyst. Then the mould filled with precursor was placed at high temperature until the precursor got hard to be solid. Thirdly, the striped precursor was placed in a furnace for sintering above $1000^{\circ} \mathrm{C}$ to make the ceramic structure dense and harder. Fourthly, ceramic fine machining, for example, cutting, milling, and polishing, was carried out to fabricate the sensor substrate as our designed dimension. Fifthly, silver was printed on the inner wall of the cavity and specific antenna pattern was printed on the cap as we have designed. It needs to be noted that the thickness of the printed silver should be no less than 12 um to prevent skin effect at high frequency. Then the silver printed substrate was placed in furnace for sintering up to $850^{\circ} \mathrm{C}$. The cap was fabricated by the same process as above. Finally, the sensor cap and substrate were bonded together through high temperature welding method, that is, melting the silver between the contact surface and then cooling it to be hard. The fabrication process of the sensor after fine machining is shown in Figure 8.

\section{Measurement of the Sensor}

To verify the validity and sensitivity of the fabricated sensor, the sensor was firstly measured in a metal-hermetic tank with uniform nitrogen pressure load as shown in Figure 9. A PCB based CPW (coplanar waveguide) interrogation antenna connected with a VNA (Agilent vector network analyzer 5061B) via a coaxial cable was used. In metal tank, electromagnetic waves, either transmitted signal or response signal, would be reflected by the metal wall. Therefore, a rubber electromagnetic wave absorber RAT-2G-3 mm was pasted on the inner metal wall, which can absorb incident waves in 


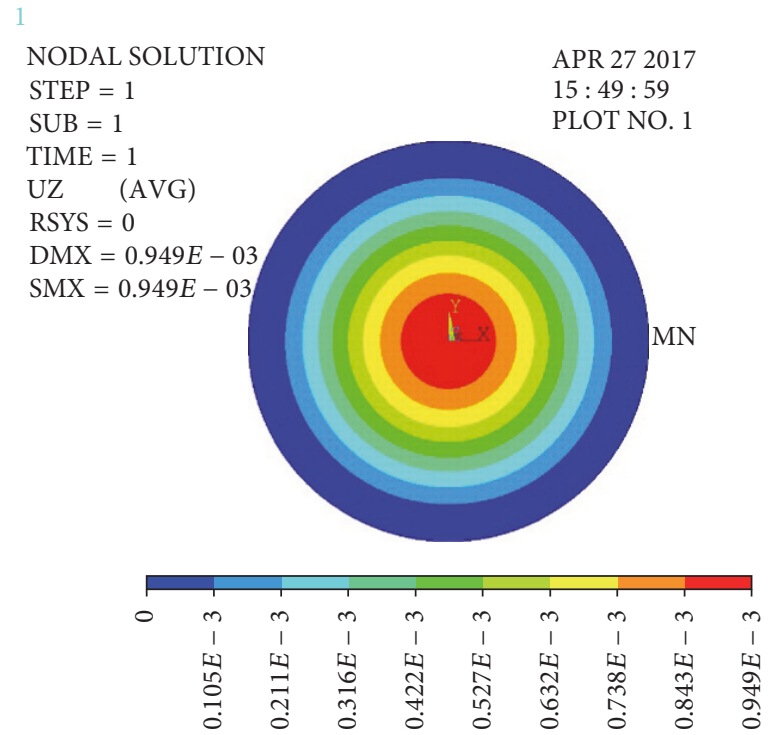

(a)

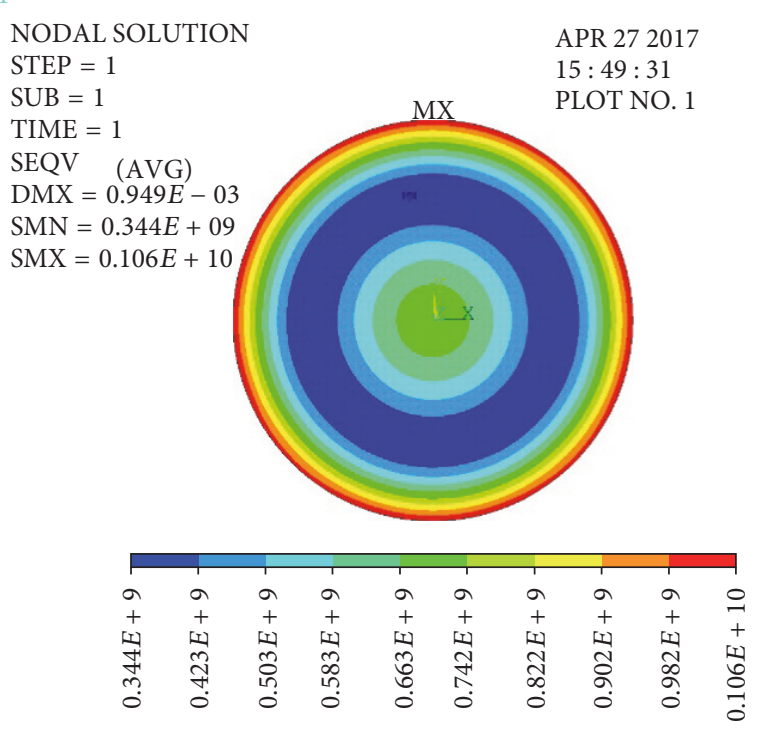

(b)

FIGURE 6: (a) Deformation distribution of the cap with 3.5 bar pressure loaded on the sensor. (b) Von Mises stress distribution.

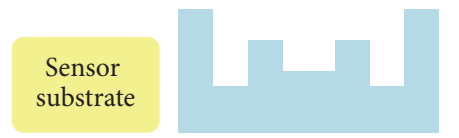

(1) Moulding

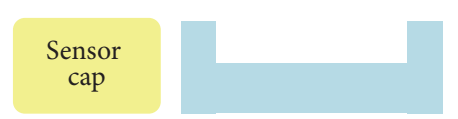

(1) Moulding

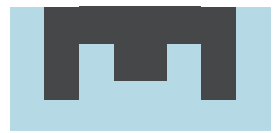

(2) Liquid ceramics Precursor filling

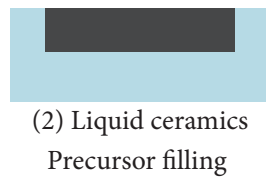

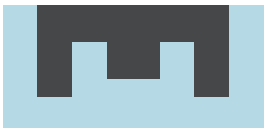

(3) Thermal insulation for solid

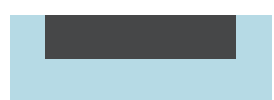

(3) Thermal insulation for solid

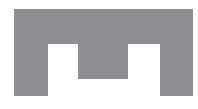

(4) Striping and sintering

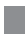

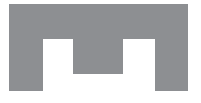

(5) Ceramic fine machining

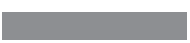

(4) Striping and sintering machining

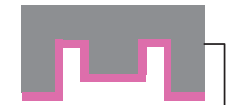

(6) Silver printing

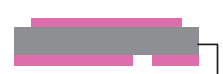

(5) Ceramic fine

(6) Silver printing

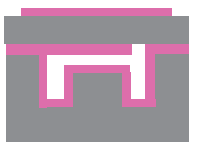

(7) Bonding

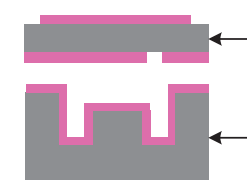

FIGURE 7: Fabrication processes of the reentrant cavity-microstrip patch antenna integrated sensor.

$2 \mathrm{GHz}$ center frequency with maximum vertical reflection of $-10 \mathrm{~dB}$. To further increase the signal-to-noise ratio (SNR) of the sensor, Time Domain Gating algorithm was used via VNA; the tested results are shown in Figure 10(a).

The pressure was loaded from 0.25 bar to 3 bar at an increased step of 0.25 bar. And the corresponding frequencies of each negative peak were extracted and plotted in Figure 10 (b). When the pressure increased from 0.25 bar to 3 bar, the resonant frequency varied from $2.03 \mathrm{GHz}$ to $1.76 \mathrm{GHz}$, presenting an approximately linear relationship with the loaded pressure. An average sensitivity of $981.8 \mathrm{kHz} / \mathrm{kPa}$ was calculated to be 50 times greater than the LC based wireless sensor proposed in [3]. But the measured resonant frequency, $2.39 \mathrm{GHz}$, of the sensor with no pressure load was deviated from the simulated frequency, $1.719 \mathrm{GHz}$; this is mainly due to the calculation error of the equivalent lumped model, the electromagnetic field distribution error caused by the punched aperture, and the process error during the fabrication.

The sensor was verified to be valid with pressure load; thus measurement at high temperature was carried on. The measurement system was shown in Figure 11. A waveguide coaxial adapter was used as an interrogation antenna to survive in higher temperature. Moreover, the operating temperature range of the absorber is $25-200^{\circ} \mathrm{C}$. It cannot survive over $200^{\circ} \mathrm{C}$. For our measurement system, there is thermal resistant material (mullite) along the round of the heat plate to make the absorber insulated with high temperature. And the environment temperature that the absorber lies in is under $100^{\circ} \mathrm{C}$ even when the heat plate is above $300^{\circ} \mathrm{C}$. The distance between the sensor and the interrogation antenna is $12 \mathrm{~mm}$. 


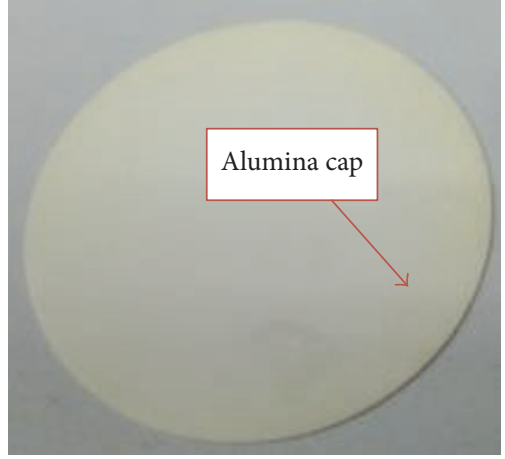

(a)

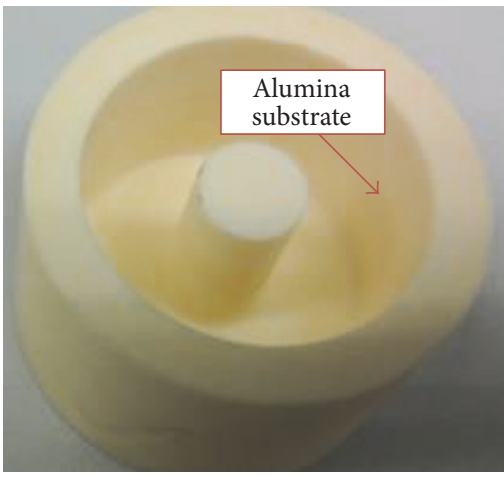

(d)

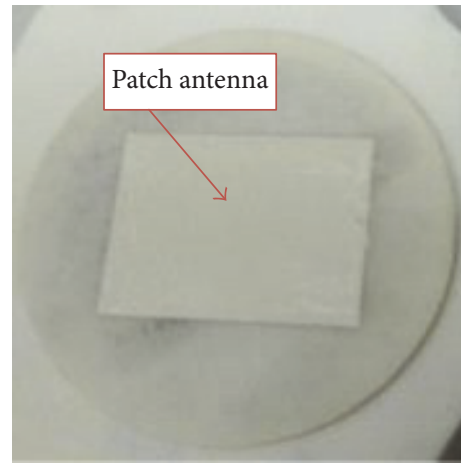

(b)

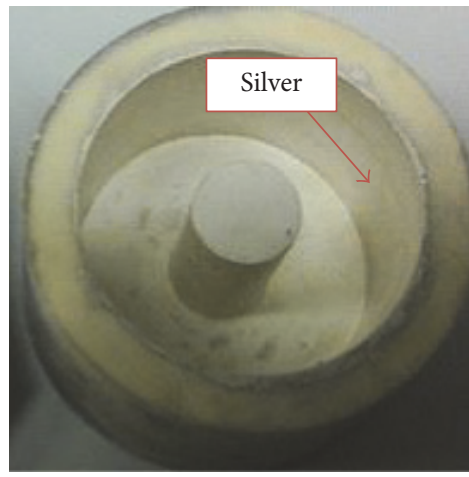

(e)

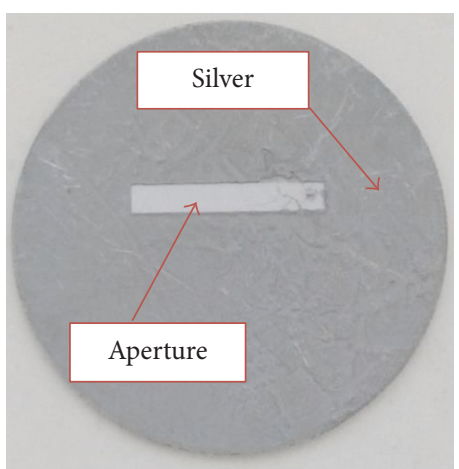

(c)

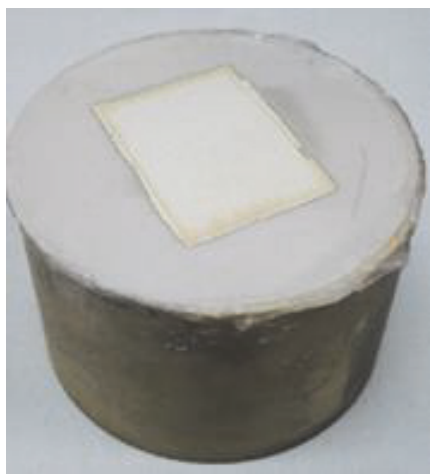

(f)

Figure 8: Sensor physical structure. (a) Sensor cap after fine machining. (b) Top view of the sensor cap. (c) Back view of the sensor cap. (d) Sensor substrate after fine machining. (e) Sensor substrate with silver printed in the inner wall. (f) Assembled sensor.

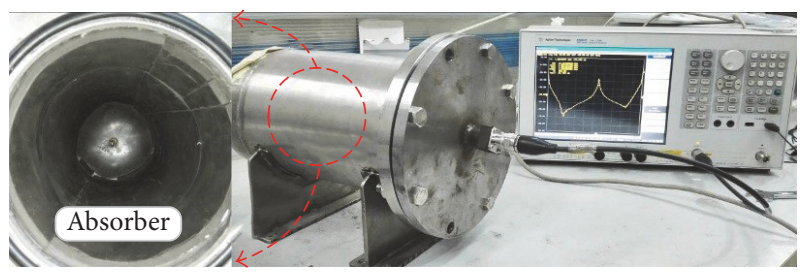

FIGURE 9: System for sensor pressure measurement at $25^{\circ} \mathrm{C}$.

Temperature in range $25-300^{\circ} \mathrm{C}$ with an increasing step of $50^{\circ} \mathrm{C}$ was set for six phases. For every phase, temperature increasing time was 15 mins and the holding time was 10 mins. During the holding time, the sensor was loaded with gas pressure from 60 to $120 \mathrm{kPa}$ at a step of $20 \mathrm{kPa}$. The measured curves for resonant frequency versus $S(1,1)$ were plotted in Figures 12(a) and $12(\mathrm{~b})$ at $25^{\circ} \mathrm{C}$ and $300^{\circ} \mathrm{C}$, respectively. We can see that the resonant frequency decreased with the increase of the pressure when the temperature was constant. The tendency was still obvious up to $300^{\circ} \mathrm{C}$. Then the resonant frequencies at different temperatures and under different pressures were extracted and plotted in Figure 12(c). For a constant temperature, it showed an approximately linear relationship between the pressure and the measured resonant frequency, proving the sensor to be effective under high temperature. The linear fitted slopes and intercepts of
TABLE 3: Linear fitted slopes and intercepts of the measured pressure at different temperatures.

\begin{tabular}{lcc}
\hline Temperature $\left({ }^{\circ} \mathrm{C}\right)$ & Slope $(\mathrm{GHz} / \mathrm{kPa})$ & Intercept $(\mathrm{GHz})$ \\
\hline 25 & -0.17 & 2.4063 \\
100 & -0.17875 & 2.4144 \\
150 & -0.18 & 2.42364 \\
200 & -0.15625 & 2.43288 \\
250 & -0.11063 & 2.43274 \\
300 & -0.1225 & 2.43765 \\
\hline
\end{tabular}

the measured curves at different temperatures are listed in Table 3.

The function relation between the slopes and the temperatures is quantic-polynomial-fitted as

$$
S=A_{0}+A_{1} T^{1}+A_{2} T^{2}+A_{3} T^{3}+A_{4} T^{4}+A_{5} T^{5},
$$

where $T$ is the temperature, $S$ are slopes in Figure 12(c), $A_{0}=$ $-0.15788, A_{1}=-8.12415 \times 10^{-4}, A_{2}=1.72278 \times 10^{-5}, A_{3}=$ $-1.85942 \times 10^{-7}, A_{4}=8.79068 \times 10^{-10}$, and $A_{5}=-1.38742 \times$ $10^{-12}$.

However, the function relation between intercepts and temperatures is quartic-polynomial-fitted as

$$
I=B_{0}+B_{1} T^{1}+B_{2} T^{2}+B_{3} T^{3}+B_{4} T^{4},
$$




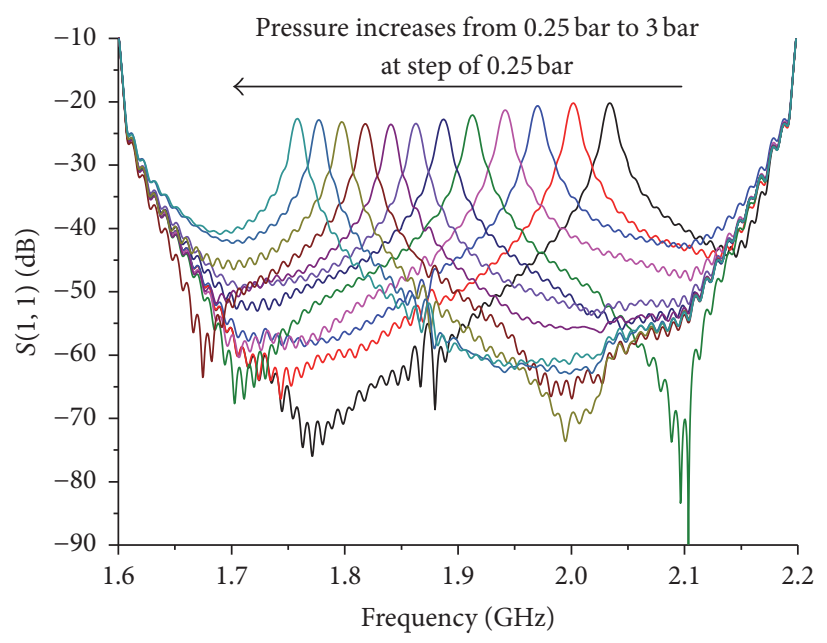

(a)

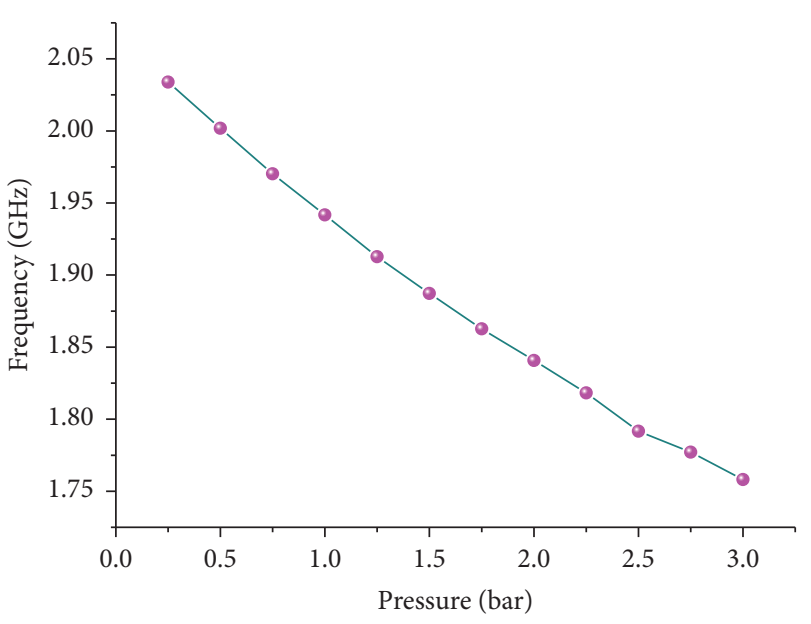

(b)

Figure 10: (a) Frequency variation with pressure from 0.25 bar to 3 bar at $25^{\circ} \mathrm{C}$. (b) Extracted resonant frequencies under different pressure at $25^{\circ} \mathrm{C}$.
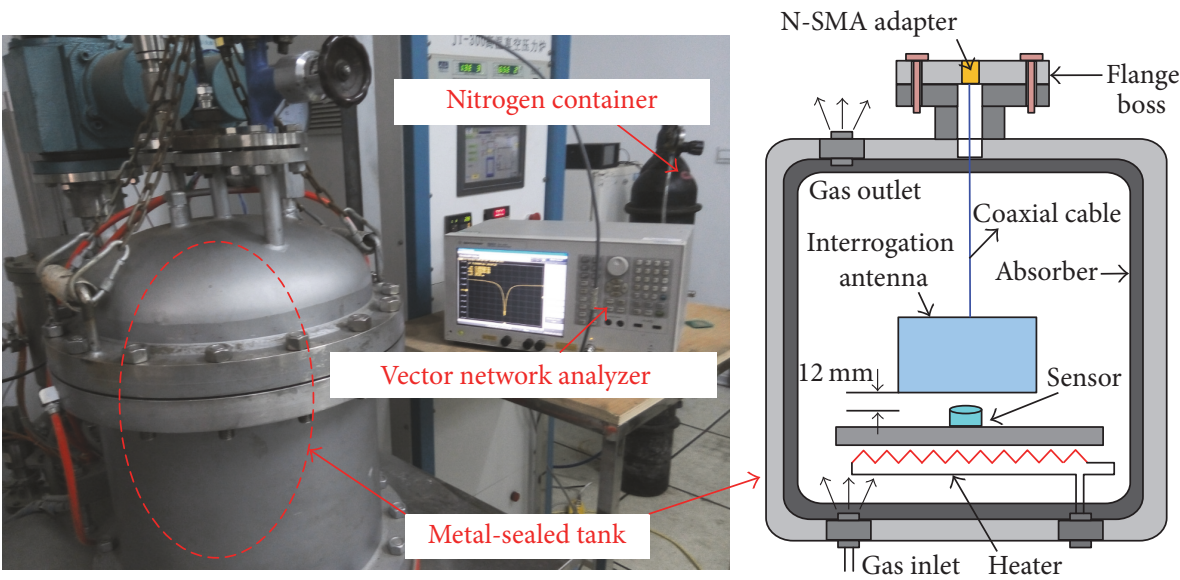

FIGURE 11: System for sensor pressure measurement at high temperature.

where $I$ represents intercepts in Figure 12(c), $B_{0}=2.41373$, $B_{1}=-4.60615 \times 10^{-4}, B_{2}=7.41625 \times 10^{-6}, B_{3}=-3.25863 \times$ $10^{-8}$, and $B_{4}=4.6213 \times 10^{-11}$.

We can see that there is a one-to-one relationship between each temperature and the correspondent linear function which can be determined using (10) and (11). For practical application, the pressure can be obtained relying on a method called table lookup on the premise that the temperature is measured in advance via methods proposed in [3-5]. It can be seen from Figure 12(d) that the pressure obtained by table lookup is around the referenced pressure with a maximum error of $5.78 \%$. It should be noted that the problem of seal between the sensor cap and substrate is one of the key factors to realize higher temperature measurement for pressure. In this paper, alumina ceramic is able to survive up to $1500^{\circ} \mathrm{C}$ and silver $900^{\circ} \mathrm{C}$. But here we did not utilize them to the temperature limitation due to the fact that the melting sealing method can just realize surface physical bonding for silver-ceramic contact, while, in temperature above $300^{\circ} \mathrm{C}$, the difference of thermal expansion coefficient between the two materials is even greater, leading to mismatch in stress; thus the melting welding surface cannot be stably sealed. This is adverse to the gas pressure loading for our contactless measurement. Consequently, our further research will be focused on the settlement of sealing problem.

\section{Conclusions}

The reentrant cavity-microstrip patch antenna integrated pressure sensor based on alumina ceramic presented in this paper is used for wireless telemetry at high temperature. Both the sensor distribution model and equivalent lumped circuit model were analyzed for the design of the sensor dimension. For measurement, Time Domain Gating algorithm was used via VNA in process of the sensor signal, effectively raising the SNR of the sensor response signal. The fabricated sensor was operated properly with gas pressure loaded up to 3 bar 


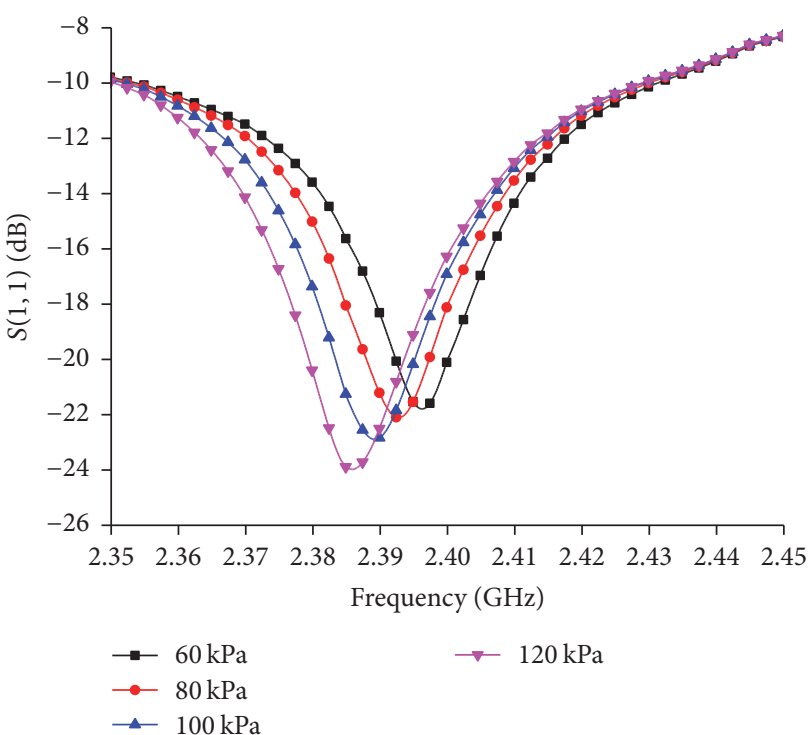

(a)

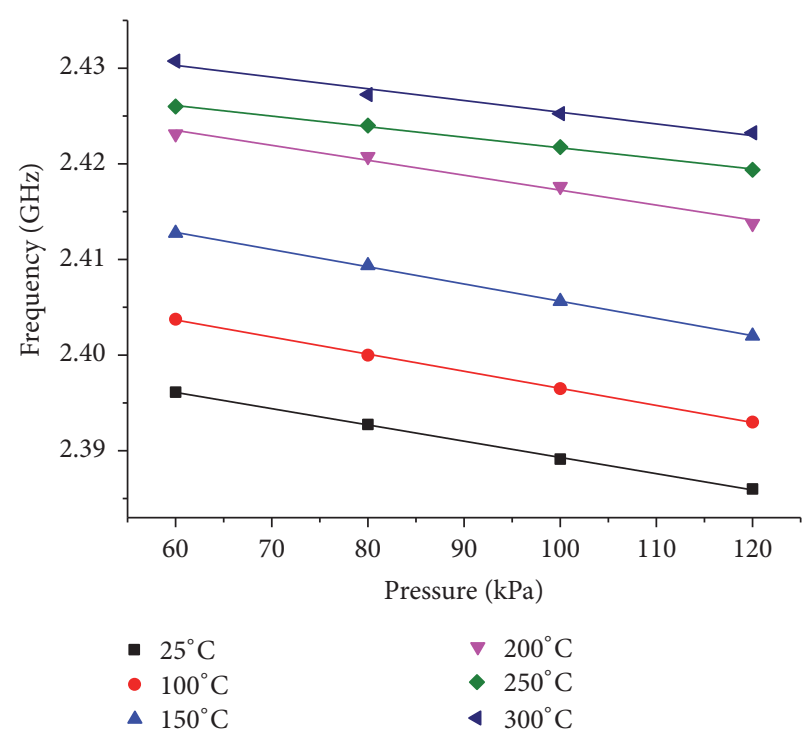

(c)

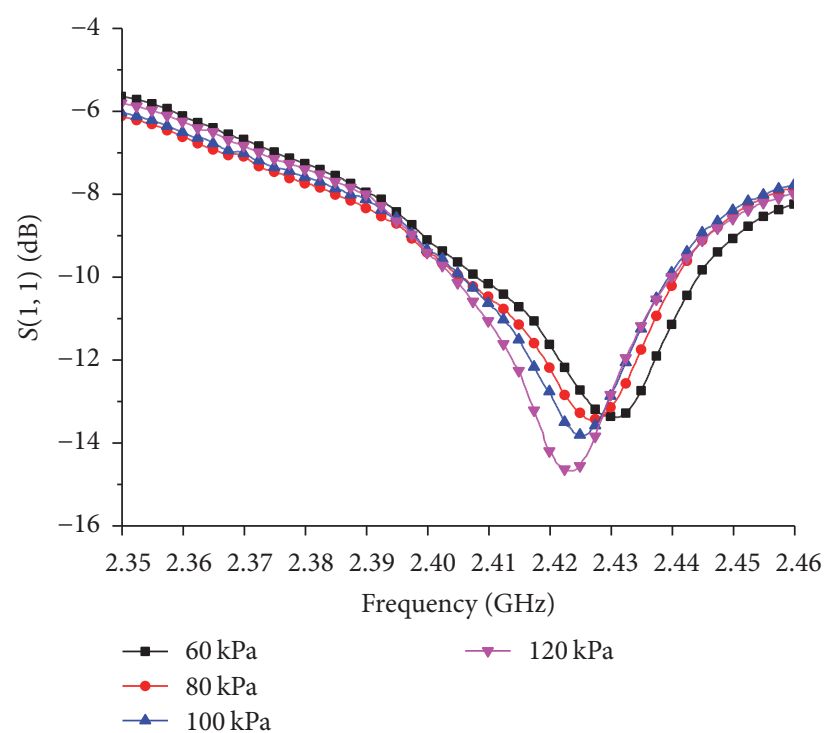

(b)

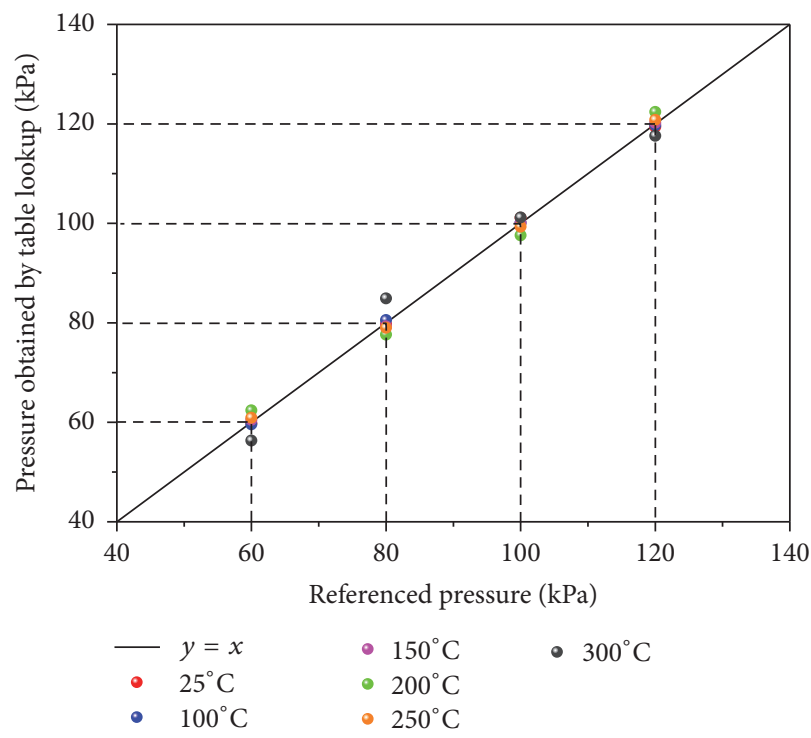

(d)

Figure 12: (a) Resonant frequency versus $S(1,1)$ under pressure of $60-120 \mathrm{kPa}$ at $25^{\circ} \mathrm{C}$ and (b) $300^{\circ} \mathrm{C}$. (c) Pressure versus resonant frequency at temperature from $25^{\circ} \mathrm{C}$ to $300^{\circ} \mathrm{C}$. (d) Referenced pressure versus pressure obtained by table lookup.

at $25^{\circ} \mathrm{C}$ with average sensitivity of $981.8 \mathrm{kHz} / \mathrm{kPa}$, proving the validity of the pressure sensor structure. And for high temperature measurement, the sensor was tested under pressure of $60-120 \mathrm{kPa}$ in temperature range of $25-300^{\circ} \mathrm{C}$ with maximum pressure sensitivity of $179.2 \mathrm{kHz} / \mathrm{kPa}$. For further improvement of the sensor properties, for example, ability of higher temperature resistance and higher operation stability, the sealing problem under high temperature needs to urgently be solved in the future. For instance, rapidly developed HTCC technology is promising to be used later for its advantages of high integration in structure and makes it easy to realize a hermetic cavity structure. This is meaningful for future pressure telemetry in situ under harsh environment.

\section{Conflicts of Interest}

The authors declare that there are no conflicts of interest regarding the publication of this article.

\section{Authors' Contributions}

All works with relation to this paper have been accomplished by all the authors' efforts. The idea and design of the sensor were proposed by Fei Lu and Yanjie Guo. Qiulin Tan and Tanyong Wei have helped fabricate the sensor. The experiments of the sensor were completed with the help of Guozhu Wu and Haixing Wang. Lei Zhang and Xiaowei Guo have proposed valuable suggestions for the manuscript. 
Jijun Xiong has helped revise the manuscript. All the authors have participated in the revision of the manuscript. In addition, Haiqun Liao from the School of Humanities and Social Sciences, North University of China, helped revise English expressions for the final manuscript. All the authors appreciate her help.

\section{Acknowledgments}

This work was supported by the National Natural Science Foundation of China (no. 61471324 and no. 51425505), the Program for the Top Young Academic Leaders of Higher Learning Institutions of Shanxi Province, China, and the Outstanding Youth Talents Program of Shanxi Province.

\section{References}

[1] M. P. Boyce, "Control Systems and Instrumentation," in Gas Turbine Engineering Handbook, vol. 19, pp. 721-768, 4th edition, 2012.

[2] M. A. Fonseca, Polymer/ceramic wireless MEMS pressure sensors for harsh environments: High temperature and biomedical applications [Dissertation], 2007.

[3] Q. Tan, T. Luo, T. Wei, J. Liu, L. Lin, and J. Xiong, "A Wireless Passive Pressure and Temperature Sensor via a Dual LC Resonant Circuit in Harsh Environments," Journal of Microelectromechanical Systems, vol. 26, no. 2, pp. 351-356, 2017.

[4] J. Xiong, G. Wu, Q. Tan et al., "Dielectrically-loaded cylindrical resonator-based wireless passive high-temperature sensor," Sensors, vol. 16, no. 12, article 2037, 2016.

[5] Q. Tan, T. Wei, X. Chen et al., "Antenna-resonator integrated wireless passive temperature sensor based on low-temperature co-fired ceramic for harsh environment," Sensors and Actuators A: Physical, vol. 236, pp. 299-308, 2015.

[6] H. Fritze, "High-temperature piezoelectric crystals and devices," Journal of Electroceramics, vol. 26, no. 1-4, pp. 122-161, 2011.

[7] R. Fachberger, G. Bruckner, R. Hauser, C. Ruppel, J. Biniasch, and L. Reindl, "Properties of radio frequency Rayleigh waves on Langasite at elevated temperatures," in Proceedings of the 2004 IEEE Ultrasonics Symposium, pp. 1223-1226, August 2004.

[8] H. Cheng, S. Ebadi, X. Ren, and X. Gong, "Wireless passive high-temperature sensor based on multifunctional reflective patch antenna up to 1050 degrees centigrade," Sensors and Actuators A: Physical, vol. 222, pp. 204-211, 2015.

[9] Y. Zhao, "RF evanescent-mode cavity resonator for passive wireless sensor applications," Sensors \& Actuators A Physical, vol. 1, pp. 322-328, 2010.

[10] L. Qin, D. Shen, T. Wei et al., "A wireless passive LC resonant sensor based on LTCC under high-temperature/pressure environments," Sensors, vol. 15, no. 7, pp. 16729-16739, 2015.

[11] J. G. Rodríguez-Madrid, G. F. Iriarte, O. A. Williams, and F. Calle, "High precision pressure sensors based on SAW devices in the GHz range," Sensors and Actuators A: Physical, vol. 189, pp. 364-369, 2013.

[12] E. Rivier and M. E. Verg-Lapisardi, "Lumped Parameters of a Reentering Cylindrical Cavity," IEEE Transactions on Microwave Theory and Techniques, vol. 19, no. 3, pp. 309-314, 1971.

[13] M. Jaworski, "On the Resonant Frequency of a Reentrant Cavity Cylindrical," IEEE Transactions on Microwave Theory and Techniques, vol. 26, no. 4, pp. 256-260, 1978.
[14] D. Peroulis et al., "Tunable cavity resonator having a post and variable capacitive coupling," US9325052, 2016.

[15] J.-M. Le Floch, Y. Fan, M. Aubourg et al., "Rigorous analysis of highly tunable cylindrical transverse magnetic mode re-entrant cavities," Review of Scientific Instruments, vol. 84, no. 12, Article ID 125114, 2013.

[16] K. Fujisawa, "General Treatment of Klystron Resonant Cavities," IRE Transactions on Microwave Theory and Techniques, vol. 6, no. 4, pp. 344-358, 1958.

[17] J. J. Barroso, P. J. Castro, J. P. L. Neto, and O. D. Aguiar, "Analysis and simulation of reentrant cylindrical cavities," International Journal of Infrared and Millimeter Waves, vol. 26, no. 8, pp. 10711083, 2005.

[18] S. Brodetsky, "Theory of plates and shells," Studies in Mathematics Its Applications Elsevier Amsterdam, vol. 3760, article 606, 1959. 


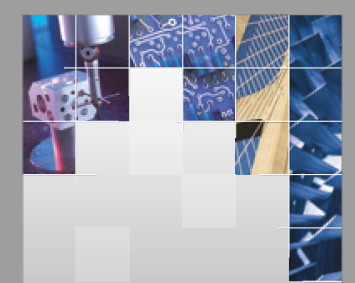

\section{Enfincering}
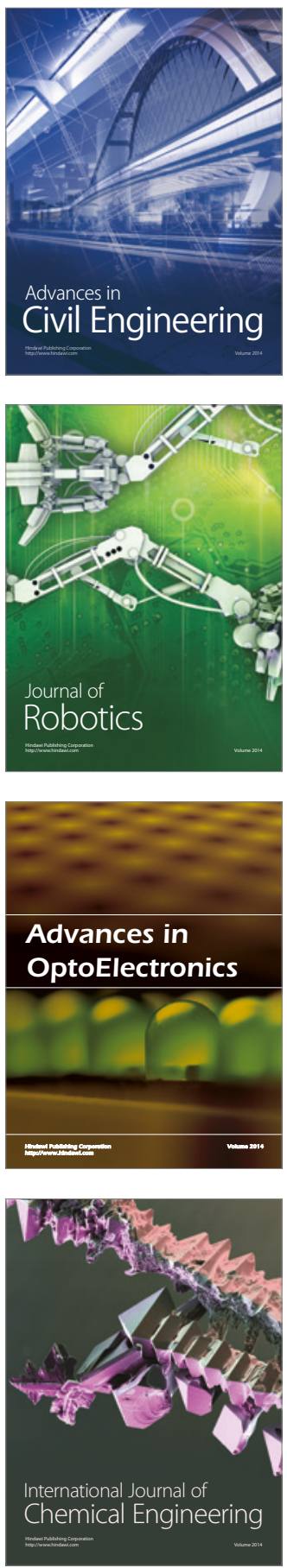

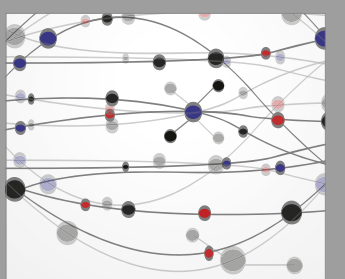

The Scientific World Journal

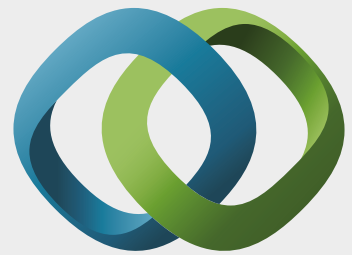

\section{Hindawi}

Submit your manuscripts at

https://www.hindawi.com
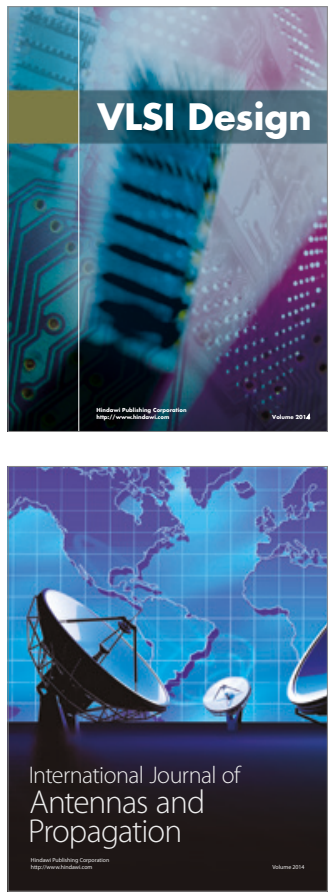

\section{Rotating}

Machinery
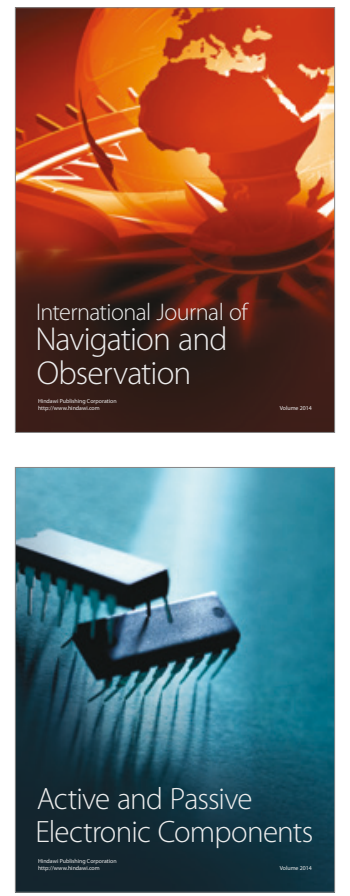
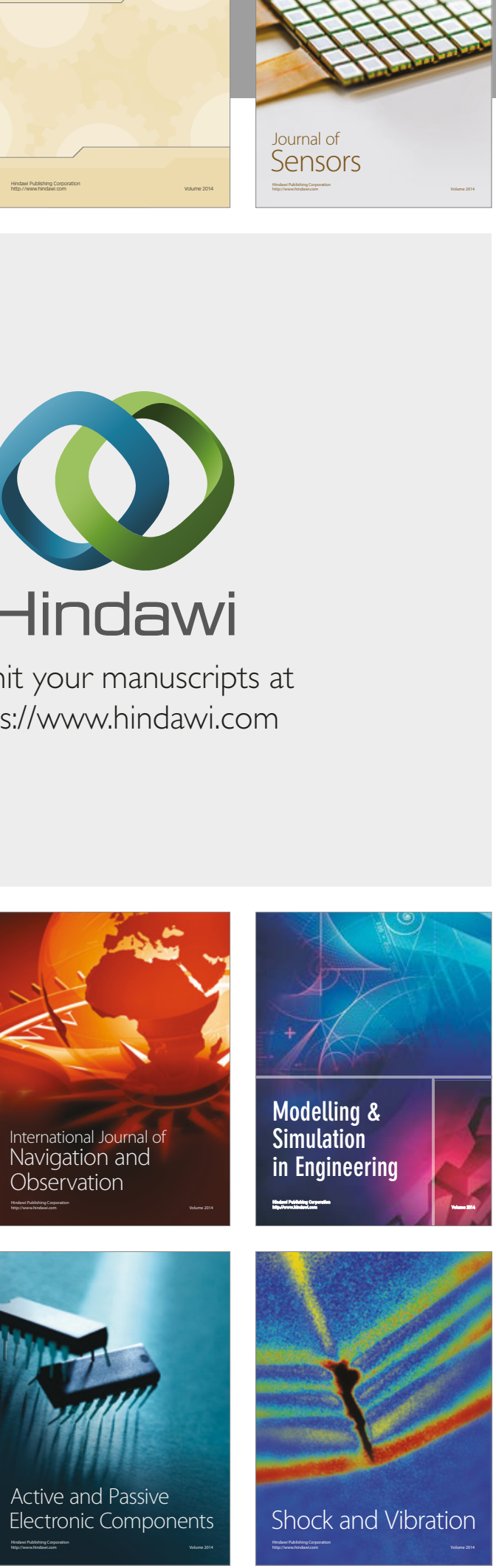
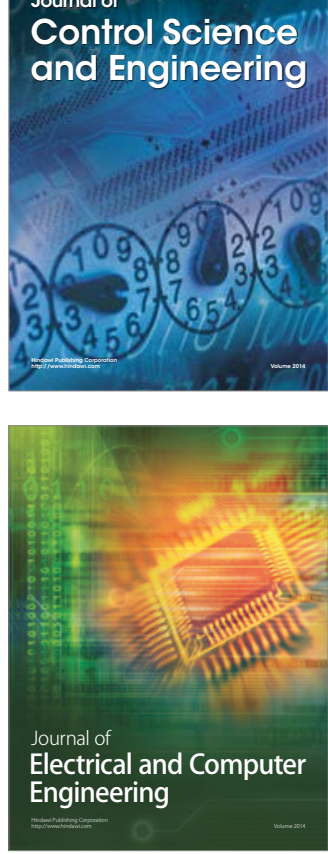

Distributed

Journal of

Control Science

and Engineering
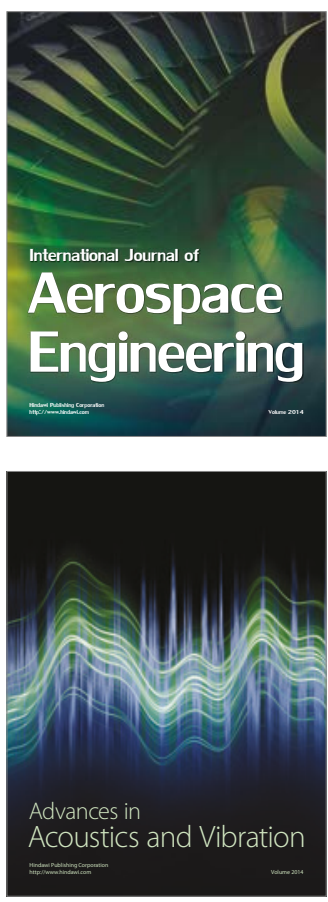

Sensor Networks 